\title{
High Prevalence of Chlamydia Trachomatis in the Sperm of Males with Low Sperm Count in Nigeria
}

\section{Okoror $\mathrm{LE}^{1 *}$ and Agbonlahor $\mathrm{DE}^{2}$}

${ }^{1}$ Department of Microbiology, Joseph Ayo Babalola University, Ikeji-Arekeji, Ilesha, Osun State, Nigeria

${ }^{2}$ Lahor Public Health, Ikpoba Hill, Benin City, Edo State, Nigeria

\begin{abstract}
Background: Chlamydia trachomatis has been linked with female infertility in male infertility and sequelae especially in developing countries which is the rationale for this study. This study view Chlamydia trachomatis as causing low sperm count in males which is a factor of male fertility.

Methods: A total of 666 sperm samples were collected from males with low sperm count and another 666 from normal individuals to serve as control. Blood samples $(n=855)$ were collected from cases and control. The sperm samples were analysed using the WHO parameters of 2010. Sperm samples were stained using the Giemsa technique. Sperm were analysed for Chlamydia trachomatis using the Polymerase Chain Reaction (PCR). The presence of other microorganisms likely to be incriminated with low sperm count was detected by culture unto nutrient agar plates, blood agar plates and Thayer Martins medium. Any sample containing any microorganism capable of causing low sperm count was eliminated from the study. The blood samples were analysed using the microimmunofluorescence assay for Chlamydia trachomatis IgM.

Results: Of the 666 cases only 417(62.6\%) were positive while the control had only 225 (33.3) positive. There was significant difference between the cases and control and they both varied in the same direction statistically. The result from the IgM Chlamydia trachomatis antibody also gave a significant difference in both cases and control. The variation between ages was not significant except in older ages of both cases and control. The 582 semen samples positive for both Chlamydia trachomatis culture and IgM analysed for WHO sperm count parameters, 432 of the cases fell short of the normal sperm count along with 150 of the controls.
\end{abstract}

Conclusion: This study then confirms Chlamydia trachomatis as a major cause of low sperm count and recommends the screening for Chlamydia trachomatis in cases of low sperm counts.

Keywords: Chlamydia trachomatis; Low sperm count; IgM; PCR; Leucocytes; Male infertility

\section{Introduction}

Chlamydia trachomatis infections are the commonest Chlamydia infection that a mere mention of Chlamydia is often referred to as disease caused by Chlamydia trachomatis. Chlamydia trachomatis are mainly spread through sexual contact and most time neonatal which have been reported to be the commonest sexually transmitted diseases in European countries and the USA [1-3] with paucity of reports from sub-saharan Africa [4]. Reports have it that the [1,2] major impact is on female genital tract, Chlamydia trachomatis could also cause urethritis, prostitis, infertility and Reiter'syndrome in males [4]. In general it is assumed that the effect of Chlamydia trachomatis female reproduction is more important than in male chlamydial infection [5]. In attempt to link male reproductive problems, a number of studies have investigated the relationship between infection and semen quality. Although some studies have shown that infection is associated with poorer semen quality [6]. Some also claimed the contrary [7]. However, it is difficult to compare all these studies hence more studies from different regions of the world is required to compliment earlier reports. The difficulty in the analysis of differences from different studies tends to be from the methods employed by these studies in analysis of Chlamydia trachomatis as well as techniques used in semen analysis which are not comparable [8]. Despite all these discrepancies, there has been a report stating that the function of human spermatozoa is significantly affected by direct exposure to Chlamydia trachomatis [9]. Other evidences have focused on epididymitis, which in young men is attributed to Chlamydia trachomatis $[10,6]$. Epididymitis is important because fertility might be affected due to the inflammation and obstruction, especially where both testis are affected [6]. The physical blockage created by obstruct the movement of sperm, C. trachomatis can also cause epithelial damage that reduces spermatogenesis, induces immunological responses to destroy or hinder sperm, and reduces the female partner's fertility [11] Reports have it that C. trachomatis may attach to spermatozoa thereby immobilizing them [12]. The importance of C. trachomatis in causing sterility have been reported to be of public health consequences for various studies from developed countries [13] as compared to developing countries where there is paucity of reports on C. trachomatis.

The importance of methodology in screening Chlamydia trachomatis as asymptomatic infection of the male urogenital tract are difficult to detect. Screening with classical cell culture requires specimen from endo-urethra swabs [13] which are cumbersome in male asymptomatic infections and may affect the result of such screening. Gdoura et al. [13] reports that cell culture cannot be used for semen and urine samples owing to cytotoxicity, and is not very sensitive to rule out infections of accessory glands [14]. Gdoura et al. [13] also reported that immunoassays for Chlamydia may have a reduced sensitivity in asymptomatic

*Corresponding author: Okoror LE, Department of Microbiology, Joseph Ayo Babalola University, llesha, Osun State, Nigeria, E-mail: LEOkoror@gmail.com

Received April 09, 2012; Accepted May 09 , 2012; Published May 14, 2012

Citation: Okoror LE, Agbonlahor DE (2012) High Prevalence of Chlamydia Trachomatis in the Sperm of Males with Low Sperm Count in Nigeria. J Med Microb Diagn 1:108. doi:10.4172/2161-0703.1000108

Copyright: (c) 2012 Okoror LE, et al. This is an open-access article distributed under the terms of the Creative Commons Attribution License, which permits unrestricted use, distribution, and reproduction in any medium, provided the original author and source are credited. 
individuals. This different method used by different reports may have been responsible for discrepancies in reports. Hence, more studies are required especially from developing countries and such studies should be compared to those from developed countries for proper inference as to whether the infections have been under reported. A combination of methods in screening for Chlamydia trachomatis should also be used in researches.

\section{Materials and Methods}

\section{Samples}

Samples were collected from consenting individuals visiting various clinical laboratories in Nigeria for sperm count $(n=666)$ and blood ( $\mathrm{n}=855)$ (Cases). The sample size was calculated using the sample size calculator at survey systems based on average figure from the national population census of 2001 with an interval of $5(\mathrm{CI}=99 \%)$. However the blood samples were more than the sperm samples since more of the subjects were willing to submit blood samples than semen. The same number of control samples was collected from those willing subjects who have never had any case of infertility or visited hospitals for infertility diagnosis or had any form of complication relating to sperm count (Control). The criterion for inclusion in the study is that subjects will not have ejaculated within 2 to 5 days as well as not been positive to any infection of urogenitals. All the samples were tested immediately in the laboratories in any of the stop-stations created across the six geopolitical zones of the country. Sperm was collected into sterile containers by first washing the penile shaft with soap and water, the subjects were then made to masturbate themselves and collect the samples aseptically. The sperm were then analyzed immediately in the laboratory. Blood samples were collected aseptically from each of the subjects with sterile syringes into sterile vacutainers and were centrifuged (hettich) at 3000rpm; sera were collected into sterile containers and stored at $-20 \mathrm{oC}$ until analyzed.

\section{Preparation of antigens for immunofluorescence}

Antigens were prepared as culture of endocervical swabs from positive women in yolk sac of chick's embryonated eggs. The eggs were harvested after 15 days of incubation, and smears made on clean grease free slide and tested for positivity by observing for characteristic Chlamydia trachomatis inclusion bodies using the Romanowsky-Giemsa staining technique [15]. The positive ones were harvested and used as life antigens. The antigens were titrated to the required concentration before been used for immunofluorescence assay. The antigen was compared to a standard synthetic polypeptide to antigen to Chlamydia trachomatis which targeted $\operatorname{IgA}, \operatorname{IgG}$ and $\operatorname{IgM}$ and were antigens were synthetic peptides, derived from species-specific epitopes in the variable domain IV of the major

outer membrane protein (MOMP) (Lab systems, Finland) and recombinant polypeptide encoded by open reading frame 3 of the plasmid (pgp3) which was previously used by Bas et al. [16]. Specificity and sensitivity were calculated using the method previously described by Bas et al. [17].

\section{Sample processing}

The semen was taken to the laboratories at the body temperature for immediate analysis at least within one hour after ejaculation. The semen were observed for volume, $\mathrm{pH}$ and sperm count including the number of sperm or concentration, motility, and morphology, white blood cells (WBC) count. All the semen analysis followed the WHO criteria 2010. Leucocytes count was carried out by first observing a drop of semen on a clean grease free slide on a microscope and then air-fixing a spread of semen and the staining with Giemsa stain before observing under high power microscope. This was also done to observe the sperm as well, to see if they had Chlamydia trachomatis attached to them. Sperm count was done by putting a drop of semen on a clean grease free slide, covered with cover slip and observed under the microscope. The sera were brought out of the $-20 \mathrm{oC}$ refrigerator, allowed to attain room temperature and inactivated at $560 \mathrm{C}$ before being tested for immunoflourescence assay. Polymerase Chain Reaction (PCR) was also carried out on all the samples.

\section{Microimmunofluorescence Assay (MIFA)}

The MIFA was carried out using the method of Krivoshen [15] using IgM slides (Bioscience) and test carried out as directed by the manufacturer. The test was carried out to detect IgM and IgG specific to Chlamydia trachomatis. The test slides were observed under the fluorescence microscope.

\section{Polymerase Chain Reaction}

Polymerase chain reaction (PCR) was carried out as by first extracting DNA from the sperm samples and carried out as described by Gdoura et al. [13]. Chlamydia trachomatis detection using PCR was done using the forward primer CT1 "CTGAAACGTTGAGAACCACCATCTGAACCAGTATTACCTGAAAACAAC" and backward primer CT2 "GACTTTGCAACTCTTGGTGGTAGACTTGGTCATAATGGACTTTTGTTG”.

\section{Statistical analysis}

Regression analysis using the Chlamydia trachomatis positivity as dependent variable and all other factors as explanatory variable was carried out in SPSS statistical software version 17. Also where necessary Fisher's ANOVA was performed as well as the general linear model and paired $t$ test.

\section{Results}

Of the total 666 sperm samples collected from males attending diagnostic clinics in Nigeria for low sperm count 417 (62.6\%) were positive to Chlamydia trachomatis (Table 1) while the 666 control samples had only $225(33.3 \%)$ positive (Table 2 ) using the PCR. The distribution of

\begin{tabular}{|lllc|}
\hline Age groups & Average Sperm & Number Positive $(\%)$ & Total \\
\hline $18-20$ & $2 \times 10^{6}$ & $21(65.6)$ & 32 \\
\hline $21-23$ & $1.5 \times 10^{6}$ & $30(75)$ & 40 \\
\hline $24-26$ & $2 \times 10^{6}$ & $31(77.5)$ & 40 \\
\hline $27-29$ & $1 \times 10^{6}$ & $40(64.5)$ & 62 \\
\hline $30-32$ & $2 \times 10^{6}$ & $51(63.8)$ & 80 \\
\hline $33-35$ & $1 \times 10^{6}$ & $58(72.5)$ & 80 \\
\hline $36-38$ & $0.5 \times 10^{6}$ & $20(48.8)$ & 41 \\
\hline $39-41$ & $1 \times 10^{6}$ & $26(68.4)$ & 38 \\
\hline $42-44$ & $2 \times 10^{6}$ & $18(60)$ & 30 \\
\hline $45-47$ & $1 \times 10^{6}$ & $15(75)$ & 20 \\
\hline $48-50$ & $2 \times 10^{6}$ & $17(85)$ & 20 \\
\hline $51-53$ & $1 \times 10^{6}$ & $19(73.1)$ & 26 \\
\hline $54-56$ & $1 \times 10^{6}$ & $21(67.7)$ & 31 \\
\hline $57-59$ & $1 \times 10^{6}$ & $18(60)$ & 30 \\
\hline $60-62$ & $0.5 \times 10^{6}$ & $7(25.9)$ & 27 \\
\hline $63-65$ & $0.5 \times 10^{6}$ & $10(27.8)$ & 36 \\
\hline $66-68$ & $1.5 \times 10^{6}$ & $15(45.5)$ & 33 \\
\hline Total & & $417(62.6)$ & 666 \\
\hline
\end{tabular}

Table 1: Distribution of Chlamydia trachomatis in males with low sperm count (Cases) Using PCR. 
Citation: Okoror LE, Agbonlahor DE (2012) High Prevalence of Chlamydia Trachomatis in the Sperm of Males with Low Sperm Count in Nigeria. J Med Microb Diagn 1:108. doi:10.4172/2161-0703.1000108

\begin{tabular}{|llll|}
\hline Age groups & Average Sperm & Number Positive (\%) & Total \\
\hline $18-20$ & $5 \times 10^{6}$ & $4(12.5)$ & 32 \\
\hline $21-23$ & $7 \times 10^{6}$ & $10(28.3)$ & 38 \\
\hline $24-26$ & $8 \times 10^{6}$ & $18(42.9)$ & 42 \\
\hline $27-29$ & $7 \times 10^{6}$ & $18(42.9)$ & 42 \\
\hline $30-32$ & $9 \times 10^{6}$ & $26(43.3)$ & 60 \\
\hline $33-35$ & $6 \times 10^{6}$ & $35(46.7)$ & 75 \\
\hline $36-38$ & $8 \times 10^{6}$ & $37(45.7)$ & 81 \\
\hline $39-41$ & $6 \times 10^{6}$ & $15(32.6)$ & 46 \\
\hline $42-44$ & $7 \times 10^{6}$ & $10(26.3)$ & 38 \\
\hline $45-47$ & $7 \times 10^{6}$ & $10(28.6)$ & 35 \\
\hline $48-50$ & $7 \times 10^{6}$ & $4(20)$ & 20 \\
\hline $51-53$ & $6 \times 10^{6}$ & $5(23.8)$ & 21 \\
\hline $54-56$ & $5 \times 10^{6}$ & $5(20)$ & 25 \\
\hline $57-59$ & $5 \times 10^{6}$ & $8(25)$ & 32 \\
\hline $60-62$ & $6 \times 10^{6}$ & $9(31)$ & 29 \\
\hline $63-65$ & $5 \times 10^{6}$ & $3(13.6)$ & 22 \\
\hline $66-68$ & $5 \times 10^{6}$ & $8(28.6)$ & 28 \\
\hline Total & & $417(62.6)$ & 666 \\
\hline
\end{tabular}

Table 2: Distribution of Chlamydia trachomatis in males with normal sperm count (Control) Using PCR

\begin{tabular}{|c|c|c|c|}
\hline \multirow[t]{2}{*}{ Age groups } & \multirow[t]{2}{*}{ Total } & \multicolumn{2}{|c|}{ Number Positive (\%) } \\
\hline & & Low sperm count & Normal sperm count \\
\hline $18-20$ & 37 & $30(81.1)$ & $10(27)$ \\
\hline $21-23$ & 52 & $49(94.2)$ & $10(19.2)$ \\
\hline $24-26$ & 86 & $78(90.7)$ & $20(25.6)$ \\
\hline $27-29$ & 80 & $72(90)$ & $30(37.5)$ \\
\hline $30-32$ & 102 & $99(97.1)$ & $33(32.4)$ \\
\hline 33-35 & 100 & $82(82)$ & $24(24)$ \\
\hline $36-38$ & 80 & 73(91.5) & $5(6.3)$ \\
\hline $39-41$ & 40 & $31(77.5)$ & $5(12.5)$ \\
\hline $42-44$ & 49 & $1(63.3)$ & $12(24.5)$ \\
\hline $45-47$ & 50 & $42(84)$ & $2(4)$ \\
\hline $48-50$ & 30 & $22(73.3)$ & 1(3.3) \\
\hline $51-53$ & 20 & $18(90)$ & $0(0)$ \\
\hline $54-56$ & 21 & $18(85.7)$ & $8(38.1)$ \\
\hline $57-59$ & 32 & $28(87.5)$ & $3(9.4)$ \\
\hline $60-62$ & 20 & $13(65)$ & $3(15)$ \\
\hline $63-65$ & 30 & $17(56.7)$ & $3(10)$ \\
\hline $66-68$ & 26 & $20(76.9)$ & $5(19.2)$ \\
\hline Total & 855 & $723(84.6)$ & $201(23.5)$ \\
\hline
\end{tabular}

Table 3: Distribution of Chlamydia trachomatis IgM in males with low and normal sperm count.

sperm count was low $(0.5 \times 106)$ in individuals with age groups $36-38$, 60-62, 63-65 while age groups 18-20, 24-26, 30-32 48-50 having the highest number of sperm count of $2 \times 106$, the control (individuals with normal sperm count have normal sperm count throughout all the age groups even when some age groups were positive for Chlamydia trachomatis using the PCR. Statistical analysis using the linear regression analysis shows that there was a significant difference between the positive cases and control $(\mathrm{t}=1.051, \mathrm{t}=7.990, \mathrm{p}=0.618,0.470$; respectively $\mathrm{CI}=99 \%)$. The one way anova test shows that there was no much variations between groups for cases $(\mathrm{F}=19.236 ; \mathrm{p}=0.50)$ while there was no variation at all within groups. Control samples also showed no significant difference between groups $(\mathrm{F}=11.064 ; \mathrm{p}=0.860)$. However, the older age groups gave more specific difference as shown in the graph of the mean plot of positive samples both for control and cases (Figure 1,2) from analysis using PCR as compared to Chlamydia trachomatis IgM. The IgM Chlamydia trachomatis antibody distribution (Figure 3)

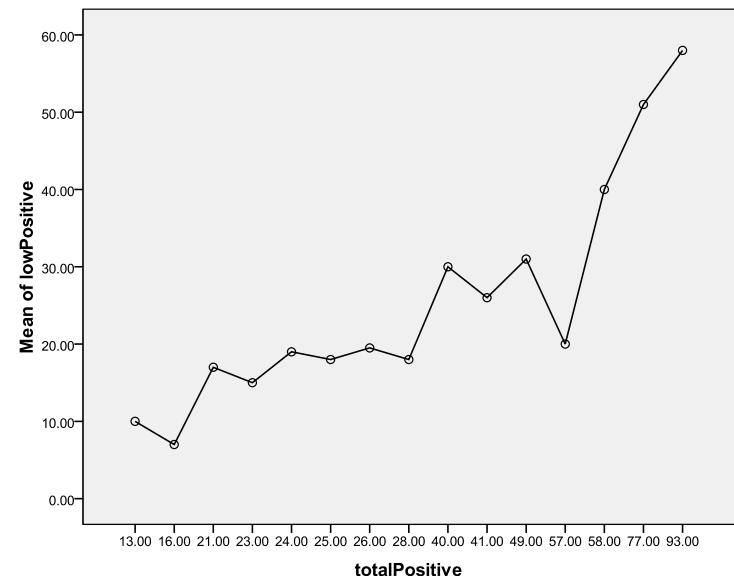

*Showing the percentage of mean of positive samples as against the total number positive for each age group which further helps in determining the differences and variation within and between the age groups.

Figure 1: Mean plot of samples from individuals with low sperm count positive for Chlamydia trachomatis from semen using PCR.

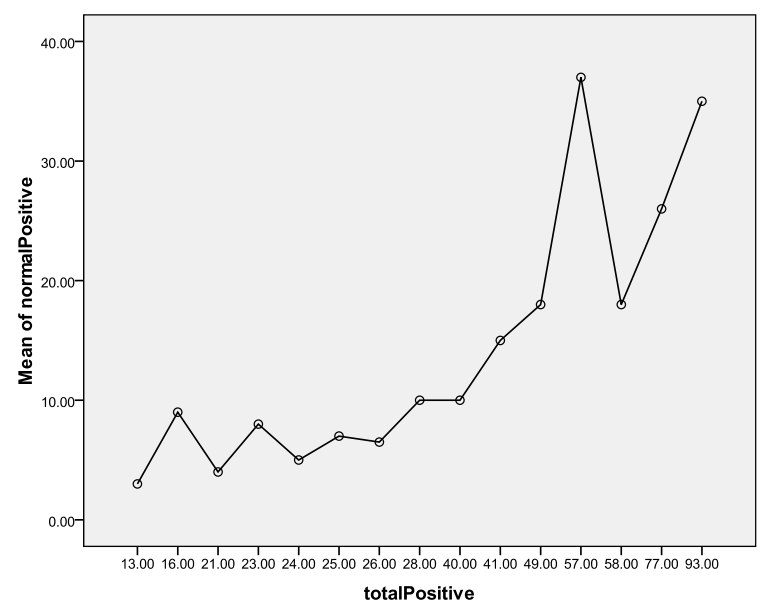

-As explained in figure 1

Figure 2: Mean plot of samples from individuals with normal sperm count positive for Chlamydia trachomatis from semen analysed using PCR.

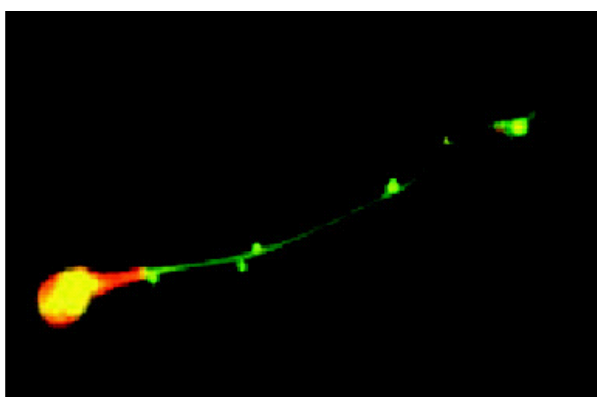

Figure 3: Chlamydia trachomatis elementary bodies attached to human sperm. From Courtney S. Hossenzadeh in Microbiology Today. In: merck manual [19].

shows that a high number of subjects with low sperm count were also positive to Chlamydia trachomatis IgM which was not significantly different from those positive from the PCR (Table 3). The control result 


\begin{tabular}{|lccccc|}
\hline Parameters & Mean & SD & SE & t & p \\
\hline Total Positive (IgM)- & & & & & \\
\hline Total Positive (PCR) & 16.59 & 19.71 & 4.78 & $3.471(2.63-30.55)$ & 0.003 \\
\hline Positive Low sperm & & & & & \\
\hline Count (IgM)-Positive & & & & & \\
\hline (PCR) & 18.00 & 17.72 & 4.30 & $4.188(5.45-30.55)$ & 0.001 \\
\hline Positive Normal Sperm & & & & & \\
\hline Count (IgM)-Positive & & & & & \\
\hline Normal (PCR) & -1.41 & 5.91 & 1.43 & $-9.86(-5.60-2.77)$ & 0.339 \\
\hline Cl=99\% & & & & & \\
\hline
\end{tabular}

infertility, it is essential to adequately exhaust the screening for pathogenic organisms and their effect on the sperm cell will help enhance this knowledge as well management.

There is very serious evidence in this study that points to the fact that Chlamydia trachomatis causes low sperm counts based on WHO specifications, and therefore infertility in males. A very high overall positive result for Chlamydia trachomatis to analysis using PCR (62.6\%) as against (33.3\%) males with normal sperm count (controls) is indicative of Chlamydia trachomatis being the cause of low sperm count since statistical analysis proved that there is significant difference between cases and controls. Several authors have reported a high incidence of Chlamydia trachomatis in both males and females [4,6,8 and 12] especially in developing countries where C. trachomatis is not normally screened for and because the pathogen is found of latent infection remains in the population unchecked. This may be the reason for high incidence even in the control subjects who are asymptomatic. The high sensitivity of PCR could also be a factor however there was no significant variation between the PCR and the IgM immunofluorescence test for all the samples (Table 4$)$. The relatively low sperm count $(0.5-2 \times 106)$ in those infected with Chlamydia trachomatis is also an evidence of the involvement of Chlamydia trachomatis in bringing down sperm counts which is in agreement with an earlier study by Cunnighan and Beagly [18]. Since the count fall short of WHO 2010 specification of 20 million counts as against an earlier 30 million counts. Other medical reports have strictly proposed a count of 40 million as being normal. Although another report has it that Chlamydia trachomatis has no effect on sperm quality [13]. Though the ANOVA test did not show any variation between the age groups for both the cases and control which opposes some earlier reports [6] but the graph of the mean plot (Figure 1,2) shows slight variation of Chlamydia trachomatis infection relative to sperm count in older ages suggesting that Chlamydia trachomatis possess more pathological problems in the sperm of older males than in that of their younger ones and this could be adduced to the fact that the graph of the mean plot was from result obtained using the PCR and as such supporting Gdoura et al. [13] which reported that PCR is more sensitive than the immunofluorescence assay. However, we suggest the slight variation could also be as a result of stronger immunity in younger age groups than in the older age groups of 51-68, which is also very visible their relatively low sperm count and opposes earlier reports [18] and which adduced this to the fact that the older men would have contacted the infection in their earlier age and due to non-treatment have invaded the sperm cells and thereby leading to this sequelae. The result from the microimmunofluorescence assay for IgM also reveal that more number of people from the younger age groups were more when compared to the older ones which also confirm that Chlamydia trachomatis is a disease of the young adults [14] but may persist and later to lead to serious sequelae in adulthood like low sperm count since the pathogen is rarely screened for in this part of the world. There was no significant difference between the result of the PCR with that of the microimmunofluorescence test for IgM thereby confirming that the IgM antibody produce against Chlamydia trachomatis, by both cases and controls is due to infection of the spermatozoa and that Chlamydia trachomatis is responsible for the low sperm count. The higher number of cases testing positive to IgM antibody to C. trachomatis, shows that most of the cases have just acquired the infection since $\operatorname{IgM}$ antibodies are a pointer to recent infection. The linear regression analysis further confirms that Chlamydia trachomatis could be responsible for the very low sperm count in cases as it shows that there was a significant difference between the cases and the control. Cases and control also vary together in the same direction as shown by their covariance analysis eliminating 
Citation: Okoror LE, Agbonlahor DE (2012) High Prevalence of Chlamydia Trachomatis in the Sperm of Males with Low Sperm Count in Nigeria. J Med Microb Diagn 1:108. doi:10.4172/2161-0703.1000108

Page 5 of 5

any form of bias in the analysis which is a further confirmation of the implication of Chlamydia trachomatis as a complication or sequelae in low sperm count. The result from both PCR of Chlamydia trachomatis and the IgM when compared using the paired t test showed that both results were consistent with each other meaning both results could infer the same thing. Hence the IgM immunofluorescence test could well serve as a good confirmatory test for the Chlamydia trachomatis especially in developing countries where resources are lacking since detection of IgM itself is a pointer to recent infection. More so when there could be PCR inhibitors in semen and may result in extra cost and time to eliminate. A combination of both methods is recommended where resources are available as no one single method is completely sufficient in analyzing this pathogen and this explains why in this study we used both test and compared both results. An observation of the parameters of low sperm count form those with low sperm count and the control reveals that more of the cases were defaulting in the standard set by WHO 2010 than the control. An example is the elevation of white blood cell (leucocytes) beyond the normal values in cases while a minimum number of the controls were defaulting. An elevation of leucocytes alone is an indication of infection. And reports have it that Chlamydia trachomatis primary infects the leucocytes and multiply within them.

This study thereby concludes that Chlamydia trachomatis is serious sequelae in male infertility and could actually be the cause of low sperm counts as observed in this study. And it is also suggested that that during semen analysis for sperm count, pathogens like Chlamydia trachomatis should also be tested for and further enhance treatment. In this study there were observations of Chlamydia trachomatis attaching to sperm cells as compared to pictures in Merck manual. And such attachment disfigures the cells and makes them unable to penetrate towards ovary fertilization. Males should also be screened as well as their female counterparts.

\section{References}

1. Baud D, Jaton K, Berteli C, Kulling JP, Greub G (2008) Low prevalence of Chlamydia trachomatis infection in asymptomatic young Swiss men. BMC Infect Dis 8: 45.

2. Manavi K (2006) A review on infection with Chlamydia trachomatis. Best Pract Res Clin Obstet Gynaecol 20: 941-951.

3. Fenton KA, Lowndes CM (2004) Recent trends in epidemiology of sexually transmitted infections in the European Union. Sex Transm Infect 80: 255-263.

4. Okoror LE, Agbonlahor DE, Esumeh FI, Umolu PI (2007) Prevalence of Chlamydia in patients attending gynaecological clinics in South Eastern Nigeria. Afr Health Sci 7: 18-24.

5. Keck C, Gerber-Schafer C, Clad A, Wilhem C, Breckwoldt M (1998) Seminal tract infections: impact on male fertility and treatment options. Hum Reprod Update 4: 891-903.

6. Kokab A, Akhondi MM, Sadeghi MR, Modarresi MH, Aarabi M, et al. (2010) Raised inflammatory makers in semen from men with asymptomatic chlamydial infection. J Androl 31: 114-120

7. Al-Mously N, Eley A (2007) Interaction of Chlamydia trachomatis serovar E with male genital tract epithelium results in secretion of proinflammatory cytokines. J Med Microbiol 56: 1025-1032.

8. Pacey AA, Eley A (2004) Chlamydia trachomatis and male infertility. Hum Fertil (Camb) 7: 271-276.

9. Eley A, Pacey AA, Galdiero M, Galdiero M, Galdiero F (2005) Can Chlamydia trachomatis directly damage your sperm? Lancet Infect Dis 5: 53-57.

10. Eley A, Oxley KM, Spencer RC, Kinghorn GR, Ben-Ahmeida ET et al. (1992) Detection of Chlamydia trachomatis by polymerase reaction in young patients with acute epididymitis. Eur J Clin Microbiol Infect Dis 11: 620-623.

11. Gonzalez GF, Munoz G, Sanchez R, Henkel R, Gallegos-Avila G, et al. (2004)
Update on the impact of Chlamydia trachomatis infection on male infertility. Andrologia 36: 1-23.

12. Okoror LE (2010) Geographical distribution/Seasonal Variation and Characterization of Chlamydia in Nigeria. VDM publishing. 189pp

13. Gdoura R, Kchaou W, Ammar-Keskes L, Charkroun N, Sallemi A, et al. (2007) Assessment of Chlamydia trachomatis, Ureplasma urealyticum, Ureplasma parvum, Mycoplasma hominis and Mycoplasma genitalium in semen and first void urine specimens of asymptomatic male partners of infertile couples. J Androl 29: 198-206.

14. Berger RE, Alexander ER, Monda GD, Ansell J, McCormick G, et al. (1978) Chlamydia trachomatis as a cause of acute "idiopathic" epididymitis. N Engl J Med 298: 301-304.

15. Krivoshein YS (1989) Handbook on Microbiology Laboratory Diagnosis of Infectious Diseases. Mir publishers Moscow. 319pp.

16. Bas S, Genevay S, Schenkel MC, Vischer TL (2002) Importance of speciesspecific antigens in the serodiagnosis of Chlamydia trachomatis reactive arthritis. Rheumatology 41: 1017-1020.

17. Bas S, Cunningham T, Kvien TK, Glennas A, Melby K, et al. (1996). The value of isotype determination of serum antibodies against Chlamydia for the diagnosis of Chlamydia reactive arthritis. Br J Rheumatol 35: 542-547.

18. Cunningham KA, Beagly KW (2008). Male genital tract Chlamydia infection: implications for pathology and infertility. Biol Reprod 79: 180-189.

19. Merck Manual Chlamydia trachomatis attachment to sperm cell 